\title{
Probability of local bifurcation type from a fixed point: A random matrix perspective
}

\author{
D. J. Albers 1 , and J. C. Sprott ${ }^{2, \text { 由 }}$ \\ ${ }^{1}$ Max Planck Institute for Mathematics in the Science, Leipzig 04103, Germany \\ ${ }^{2}$ Physics Department, University of Wisconsin, Madison, WI 53706
}

(Dated: June 27, 2018)

\begin{abstract}
Results regarding probable bifurcations from fixed points are presented in the context of general dynamical systems (real, random matrices), time-delay dynamical systems (companion matrices), and a set of mappings known for their properties as universal approximators (neural networks). The eigenvalue spectra is considered both numerically and analytically using previous work of Edelman et. al. Based upon the numerical evidence, various conjectures are presented. The conclusion is that in many circumstances, most bifurcations from fixed points of large dynamical systems will be due to complex eigenvalues. Nevertheless, surprising situations are presented for which the aforementioned conclusion is not general, e.g. real random matrices with Gaussian elements with a large positive mean and finite variance.
\end{abstract}

PACS numbers: 05.45.-a 05.45.Tp 89.75.-k 89.75.Fb

Keywords: dynamical systems, structural stability, stability conjecture, Lyapunov exponents, complex systems, routes to chaos, random matrices, bifurcation theory

\section{INTRODUCTION}

Determination of the types of random matrices that constitute a physically relevant set depends markedly on the field of study. From the perspective of those interested in quantum mechanical phenomena (e.g. nuclear physics), one might be led to believe that random matrices that are not unitary or Hermitian are of no particular physical interest [30] [33]. However, if one were interested in dynamics a la Poincaré, where an understanding of the derivative along an orbit is of utmost importance and where Lyapnov exponents are the key quantities of interest; then matrices of prime concern are real random matrices from the general linear group (the dissipative case) or the special linear group (the non-dissipative case). The interest in real random matrices arises because they form the linear portion of the derivative or the tangent space of a dynamical system at a given point along a trajectory [1] [35] 28] [32], [34]. From a dynamical reconstruction perspective, where time-series data is used [17], it is companion matrices and thus polynomials with random coefficients that are of interest. Because we are concerned with dynamical systems in general, specifically probabilities of bifurcations from fixed points and the factors that determine those probabilities, we will focus on real random matrices with various distributions followed by a practical construction using "universal approximators" with random weights.

To place the current study in context, consider first the following background. Doyon et. al. 11] argued that the most likely first bifurcation and route to chaos given a particular set of dynamical systems was that of the RuelleTakens quasi-periodic route to chaos based upon the random matrix results of Girko [19] (for other useful versions of this random matrix result see Edelman [13], and Bai [8]). Likewise, Sompolinsky et. al. [38] showed that as the dimension of a dynamical system is increased, the location of the bifurcation from a fixed point decreased toward zero; and the routes to chaos region of parameter space subject to parameter variation decreased in length. Doyon et. al. and Sompolinsky et. al. both considered neural networks with a single hidden layer (a single layer of neurons) whose input layer was entirely replaced by it's output layer at each time-step (i.e. "vector" neural networks). However, Sompolinsky et. al. considered the continuous time case where as Doyon et. al. worked in discrete time - both constructions yielded a similar set of conclusions. In previous publications [5] the authors considered time-delay neural networks such as those presented in the work of Hornik et. al. 25. that have been found to be universal approximators (i.e. they can approximate $C^{r}(r \geq 0$ mappings and their derivatives on compact, metrizible sets) and also came to similar conclusions despite the significant differences in the constructions. In [4] the authors claimed that as the dimension of the dynamical system is increased, flip, fold, or any bifurcations due to real eigenvalues, and strong resonance bifurcations will be vanishingly rare and the route to chaos from a fixed point in parameter space will consist of periodic orbits with high-period $(>4)$ and quasi-periodic orbits. The basic idea of the above

*Electronic address: albers@cse.ucdavis.edu

${ }^{\dagger}$ Electronic address: sprott@physics.wisc.edu 
arguments follows from the matrix theory of Girko [19], Edelman [13], and Bai [8] and is the following: given a square matrix whose elements are real random variables drawn from a distribution with a finite sixth moment, in the limit of infinite dimensions, the normalized spectrum (or eigenvalues) of the matrix will converge to a uniform distribution on the unit disk in the complex plane. It is worth noting that the convergence in measure is not absolutely continuous with respect to Lebesgue measure. Nevertheless, if the Jacobian of the map at the "first" bifurcation point (i.e. the bifurcation from the fixed point) is a high-dimensional matrix whose elements have a finite sixth moment, it is reasonable that the bifurcation would be of type Neimark-Sacker (via a complex eigenvalue) instead of a flip or fold (via a real eigenvalue), with probability approaching unity as the dimension goes to infinity.

Each of the studies mentioned above consist of a statistical sampling of a space of mappings via a weight structure imposed upon those mappings. In a sense, it is a statistical sampling of the effects that the weight matrices have on the dynamics. The evidence discussed in the former paragraph leads to at least three important questions: how robust are the results with respect to the measures imposed upon the weight matrices; given that there do exist observable period-doubling bifurcations in high-dimensional dynamical systems, how does this occur given the random matrix style arguments; how can these results be connected and compared with real world systems - what are the links with the natural world? Addressing the first two questions is a matter of carefully studying how the results from random matrix theory apply specifically to what has been observed computationally. Discussion of the third issue lies with providing a construction that yields comparison with real-world data.

As we will see, the distributions and perturbations of the distributions of the elements of random matrices can have profound and surprising effects on a bifurcation sequence while having negligible effects regarding the generality of the proven theorems. Thus, perturbations of the weight distributions of the computationally studied systems can be made in such a way that significantly alters the conclusions of the random matrix arguments as applied to bifurcation theory. This will provide answers to why and how period-doubling sequences can be observed in high-dimensional systems. This of course does not marginalize the results discussed above; it only qualifies them, for despite the effects we observe, the former results remain quite general.

We will begin with a general study of linear dynamical systems at fixed points. Generically, all dynamical systems at stable fixed points can be thought of as linear maps via the implicit function theorem. We will identify the Jacobian of the aforementioned dynamical systems with random matrices with various distributions. We will follow this approach, adopting the framework of random polynomials which can be likened to companion matrices. The linear part of the derivative of time-delay dynamical systems is that of a companion matrix. Thus, we will study general, linear, time-delay dynamical systems subject to weight distributions of the coefficients of the characteristic polynomial. The final framework we will use - which was begun in [5] - is that of scalar, time-delay neural networks that are commonly used to reconstruct a dynamical system from empirical, time-series data. This will begin to forge a connection with natural systems because networks such as the ones we study can be used to fit other data sets, and the weight distributions of the trained networks can be compared with those from more theoretical distributions.

\section{CONSTRUCTION}

Begin with a discrete-time, $C^{r}(r>0, r \in N)$ dynamical system that maps a compact subset $U \subset R^{d}$ to itself:

$$
F: U \rightarrow U
$$

However, due to practical motivations we will instead consider a set of functions that can universally approximate a properly chosen time-delay map of $F$ (we will discuss this more in section ЩA). Specifically, we consider $f$ to be explicitly defined:

$$
y_{t+1}=f\left(y_{t}, \ldots, y_{t-d}\right)
$$

where $f$ is $C^{r}$ and $y_{i} \in R$. In a practical sense, $f$ can be thought of as a standard feedforward neural network given by:

$$
x_{t}=\beta_{0}+\sum_{i=1}^{n} \beta_{i} \tanh \left(s \omega_{i 0}+s \sum_{j=1}^{d} \omega_{i j} x_{t-j}\right)
$$

which is a map from $R^{d}$ to $R$. Here $n$ is the number of hidden units (neurons), $d$ is the number of time lags which determines the system's input (embedding) dimension, and $s$ is a scaling factor on the connection weights $w_{i j}$. The initial condition is $\left(x_{1}, x_{2}, \ldots, x_{d}\right)$ and the state at time $t$ is $\left(x_{t}, x_{t+1}, \ldots, x_{t+d-1}\right)$. The approximation theorems of Ref. 25] and well known time-series embedding results Ref. 41] together establish an equivalence (given properly 


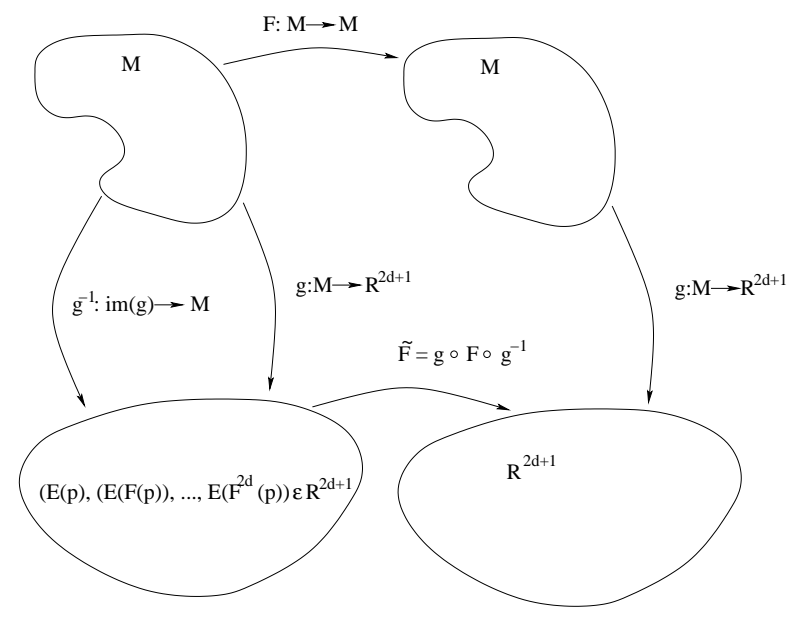

FIG. 1: Schematic diagram of the Takens embedding theorem and how it applies to our construction.

chosen weight distributions) between this class of neural networks and the general dynamical systems of interest here 3].

We sample the $(n(d+1)+1)$-dimensional parameter space taking (i) $\beta_{i} \in[0,1]$ uniformly distributed and rescaling them to satisfy $\sum_{i=1}^{n} \beta_{i}^{2}=n$, (ii) $w_{i j}$ as normally distributed with zero mean and unit variance, and (iii) the initial $x_{j} \in[-1,1]$ as uniform. We will focus largely on behavior types as a function of the parameter $s$, which can be interpreted as the standard deviation of the $w$ weight matrix, and the embedding dimension $d$. Note that for $x \approx 0$ $\tanh (x)$ is nearly linear. Thus, choosing $s$ to be small forces the dynamics to be mostly linear, yielding fixed-point behavior. Increasing $s$ yields a route to chaos [5] [4]. Due to this, $s$ provides a unique bifurcation parameter that sweeps from linear to highly nonlinear parameter regimes. In this paper we will however only consider the networks up to the first bifurcation.

Time-delay, scalar networks have an extremely convenient property that aids in the calculation of the local derivative and in the computation of the eigenvalue of the Jacobian at a fixed point; the local derivative of our scalar networks are companion matrices:

$$
D f_{x}=\left[\begin{array}{ccccccc}
a_{1} & a_{2} & a_{3} & \cdots & a_{d-2} & a_{d-1} & a_{d} \\
1 & 0 & 0 & \cdots & 0 & 0 & 0 \\
0 & 1 & 0 & \cdots & 0 & 0 & 0 \\
\vdots & \ddots & \vdots & & & & \\
0 & 0 & 0 & \cdots & 0 & 1 & 0
\end{array}\right]
$$

The $a_{k}$ 's are given as:

$$
a_{k}=\frac{\partial x_{t}}{\partial x_{t-k}}=\sum_{i=1}^{n} \beta_{i} s w_{i k} \operatorname{sech}^{2}\left(s w_{i 0}+s \sum_{j=1}^{d} w_{i j} x_{t-j}\right)
$$

\section{A. Approximation theory}

To understand how the neural networks in our study fit into the dynamical systems framework, we must consider two issues, the relationship between time-delay dynamical systems and the approximation capabilities of neural networks.

The answer to the first issue is given by Takens - and more tailored to our construction, the paper of Sauer et. al. 37] - is yes with certain constraints on $F$. Namely, the box-counting dimension of the set of periodic orbits of period $p,\left(A_{p}\right)$ must be less than $\frac{p}{2}$, and $D F_{p}$ must have distinct eigenvalues. These constraints are likely satisfied since we do not consider portions of parameter space that easily yield periodic orbits.

The relationship of general time-delay dynamical systems is given in Fig. (1) in which $F$ is a $C^{r}$ dynamical system, $E: M \rightarrow R$ a is a "measurement function," ( $E$ is a $C^{k}$ map) embedding $g: M \rightarrow R^{2 d+1}$ is explicitly given by:

$$
g\left(x_{t}\right)=\left(E\left(x_{t}\right), E\left(F\left(x_{t}\right)\right), \ldots, E\left(F^{2 d}\left(x_{t}\right)\right)\right)
$$


In a colloquial, experimental sense, $\tilde{F}$ just keeps track of the observations from the measurement function $E$, and, at each time step, shifts the newest observation into the $2 d+1$ tuple and sequentially shifts the scalar observation at time $t\left(y_{t}\right)$ of the $2 d+1$ tuple to the $t-1$ position of the $2 d+1$ tuple. In more explicit notation, $\tilde{F}$ is the following mapping:

$$
\left(y_{1}, \ldots, y_{2 d+1}\right) \mapsto\left(y_{2}, \ldots, y_{2 d+1}, g\left(F\left(g^{-1}\left(y_{1}, \ldots, y_{2 d+1}\right)\right)\right)\right)
$$

where, again, $\tilde{F}=g \circ F \circ g^{-1}$.

The response to the question regarding the approximation capabilities of neural networks is significantly more complicated due to two particular issues - the networks have only finitely many parameters and the induced measure on the weights of the neural networks determines what phenomena will be fit stably. That networks we consider have finitely many parameters implies there will never exist a one-to-one or onto correspondence between the space of $C^{r}$ diffeomorphisms and neural networks. The best that can be achieved is a density in the relevant function space with infinitely many parameters. This is a fundamental functional approximation issue that has no chance of being improved, however, this issue is clearly not terminal for our study. The more interesting problem is that of the induced measure - specifically, how the space of neural networks with the induced measure on the parameter space represent approximates of the dynamical systems that satisfy the technical restrictions of Sauer et. al. Further, what subsets of the space of $C^{r}$ dynamical systems are being selected out by such a measure (or even our measure) is a very interesting question. There surely exists a measure on the space of parameters of neural networks such that (given the dimension is high enough) the selected neural networks will approximate and correspond to a set of the $C^{r}$ mappings that satisfy the restrictions of Sauer et. al. In fact our measure often satisfies this criterion given certain restrictions. Such issues hopefully can be addressed by applying information geometry to the parameter space of neural networks following work of [6]. However, a clear understanding of what portion of the $C^{r}$ mappings we are sampling is currently difficult to discern.

Setting the issues of a finite number of parameters aside and leaving any constraints on the weights behind, the neural networks we utilize can approximate $\tilde{F}$ and its derivatives (to any order) to arbitrary accuracy [24] 25]. Precise statements of the neural network approximation theorems require machinery from functional analysis (see [2]) and is covered in detail in the papers by Hornik et. al. and is discussed in [3]. The neural networks can approximate mappings from Sobolev spaces, $S_{p}^{m}(U, \lambda)$, such as the one defined here:

Definition 1 For any positive integer $m$ and $1 \leq p<\infty$, we define a Sobolev space $S_{p}^{m}(U, \lambda)$ as the vector space on which $\|\cdot\|_{m, p}$ is a norm:

$$
S_{p}^{m}(U, \lambda)=\left\{f \in C^{m}(U)|| \mid D^{\alpha} f \|_{p, U, \lambda}<\infty \text { for all }|\alpha| \leq m\right\}
$$

Equipped with the Sobolev norm, $S_{p}^{m}$ is a Sobolev space over $U \subset R^{d}$.

Neural networks can also approximate most $C^{r}$ mappings and their derivatives. However, if the mapping is piecewise differentiable, the neural networks must be fit independently on both sides of the discontinuity. Nevertheless, neural networks form a set of "universal approximators" — they can approximate nearly any imaginable mapping.

\section{The implicit measure}

In a very practical sense, we are implicitly imposing a measure on the set of neural networks via the probability distributions of the weights on $R^{n(d+2)+1}$ (i.e. the probability distributions form a product measure on $R^{n(d+2)+1}$ ). This will introduce a bias into our results that is unavoidable in such experiments; the very act of picking networks out of the space will determine, to some extent, our results. Examples of how important this is will be revealed as we consider the numerical results. Unlike actual physical experiments, we could, in principle, prove an invariance of our results to our induced measure, however this is difficult and beyond the scope of this paper. It suffices for our purposes to note specifically what our measure is (the weight selection method), and how it might bias our results. The selection method will include all possible networks, but clearly not with the same likelihood. In the absence of a theorem with respect to an invariance of our induced measure, we must be careful in stating what our results imply about the ambient function space.

\section{B. Why scalar neural networks?}

Time-delay scalar neural networks are enticing to study because they allow for a combination of the perspectives of functional analysis, topological dynamics, and the practical, real world. From the functional analysis perspective, the 
neural networks we study are diverse universal function approximators - they can approximate nearly any mapping one might wish to approximate [25]. Thus, studying the space of neural networks represents a practical study of a common space of mappings used by time-series analysists to reconstruct unknown dynamics from time-series data 17 . [40] 27. From the perspective of topological dynamics, if one wishes to study general dynamical systems, there is always the issue of how to relate such studies to the natural world that they were originally meant to model. Studying time-delay dynamics as they relate to $C^{r}$ dynamical systems is a partial connection or link between abstract dynamics and the natural world since the natural world is often studied with time-series data 33]. Yet there is the issue of relating time-delay dynamics to a specific natural system or class of systems. However, one of the key sources of the problem of relating the space of neural networks to a space of general dynamical systems is the implied measure on the weight space. This is also where the practical link between the natural world and general time-delay dynamical systems can be found. The weight distributions form a clear link between the abstract dynamics world via embedding and function approximation of real time-series data from natural phenomena. If the dynamics that arise from the space of neural networks can be understood in terms of their weight distributions and on the neural networks can be fit to data from nature - the fit weight distributions can be compared with the dynamics dependent on the weight distributions. Thus, aside from being an extremely general class of dynamical systems and universal approximators in their own right, neural network weight distributions are a possible link between the abstract world of mathematical dynamics and the natural world.

\section{RANDOM MATRIX THEORY}

Our discussion of random matrix theory will be limited to the circular law of Girko [19], Bai [8], and Edelman 13. In general, circular laws in random matrix theory relate the distributions of elements of a random matrix to the distribution of those matrices' eigenvalues on a disk, usually centered at the origin, in the complex plane. We will begin by discussing the circular law outright and follow this with a discussion of the expected value of real eigenvalues of a random matrix, and various related results. In both of the sections that follow, all of our matrices will be $n \times n$ matrices with real elements drawn from a random distribution yet to be specified.

Dynamical systems at fixed points can be identified with random matrices via the linear derivative map of the dynamical system $f$ where each of the terms in the matrix is given by a number from a random distribution. This is quite general - given that the range and domain of the dynamical system have the same dimensions. Any generic, discrete-time, $d$-dimensional dynamical system at a fixed point can be recast as a linear map $g=A x$ where $A$ is a $d \times d$ matrix via the implicit function theorem. Thus, studying the spectrum of random matrices is, in a way, equivalent to studying bifurcations of dynamical systems at non-degenerate fixed points with respect to the variation of a linear scaling parameter; because the spectrum of $D f$ will yield the entire geometric structure of $f$.

\section{A. The Circular Law}

The study of the circular law has a long, somewhat colorful, and debated history. In the early 50's it was conjectured that the empirical spectral distribution (i.e. the distribution of eigenvalues) of $n \times n$ matrices with independent and identically distributed elements that were normalized by $\frac{1}{\sqrt{n}}$, converged to a uniform distribution on the unit disk in the complex plane. This is what is refered to as the circular law. In 1965 Ginbre [18] proved this conjecture in the case where the random matrix is complex and has elements whose real and imaginary parts are independent and normally distributed (i.e. the real and imaginary parts are independent normals). V.I. Girko published, in 1984 [19], 1994 [21], and again in 1997 23] 22], papers proving the circular law for real, random, Gaussian, matrices. Girko's circular law states that as $n \rightarrow \infty$, the distribution of $\frac{\lambda}{\sqrt{n}}$ tends to uniformity on the unit disk. This result, which implies that as $n \rightarrow \infty$, the probability of an eigenvalue being real must go to zero, is a key ingredient towards showing that local bifurcations from fixed points due to purely real eigenvalues will be unlikely. It is this result that limits the kinds of generic local bifurcations from fixed points we can observe in the infinite-dimensional limit. One particularly unfortunate problem with Girko's measure (as well as Edelman's and Bai's) is that it is not absolutely continuous with respect to Lebesgue measure with increasing but finite dimension (the infinite-dimensional limit is however absolutely continuous). In particular, the probability of an eigenvalue being real with respect to Edelman is higher than one might expect for finite dimensions. Thus, convergence in distribution to uniformity on the unit disk becomes an issue. However, Edelman [15] derived a formula for the expectation value of real eigenvalues in Girko's measure in finite dimensions (we will discuss that result in section IIIB) which we will discuss since it is very important with respect to our results. In the process of deriving this expectation formula, Edelman also proved Girko's result. In 1997, Bai [8] provided an alternate proof of the circular law for real random matrices with a significantly weaker hypotheses 
than either Edelman or Girko. Bai's result requires that the elements of the matrix be from a distribution with only a finite sixth moment. We will state Bai's result here since is it is both the simplest, and most general.

Theorem 1 (Circular law [8] (page 496)) Suppose that the entries of a $n \times n$ matrix $M$ have finite sixth moment and that the joint distribution of the real and imaginary part of the entries has a bounded density. Then, with probability 1 , the empirical distribution $\mu_{n}(x, y)$ tends to the uniform distribution over the unit disk in two-dimensional space.

Understanding the difference between the results of Bai, Girko, Edelman, the convergence of the density of eigenvalues on the unit disc, and how this is related to the eigenvalue with the largest magnitude will be the focus of sections $\mathrm{VIA}$ and $\mathrm{VIB}$

\section{B. Expected Value of Real Eigenvalues and Related Results}

The circular law will provide the intuition for the conjecture we will state shortly, but it is difficult to use for our purposes, and provides little practical understanding of how the distribution of eigenvalues evolves and converges to uniformity as the dimension of the matrix is increased. Luckily, Edelman essentially evaluated the integral formula of Girko (in spirit, at least) and arrived at a formula for the expected number of real and complex eigenvalues as a function of the dimension of the matrix. Edelman has proved the following formulas and theorems which will be useful and relevant for our work: a formula for the density of real eigenvalues in the complex plane as a function of the dimension of the matrix; a formula for the density of non-real eigenvalues on the complex plane as a function of the dimension of the matrix; a formula for the expectation value of real eigenvalues of a matrix as a function of the dimension of that matrix; and a theorem that states that the real eigenvalues converge in distribution to that of a uniform random variable on $[-1,1]$ in the limit of an infinite-dimensional matrix. For completeness, we will reproduce the aforementioned results, noting that all the statements that follow are relevant for matrices such that $A \in R^{n^{2}}$ where $a_{i j} \in N(0,1)$.

We will begin with two definitions, the true density of real eigenvalues of a real random matrix and the probability density of real eigenvalues of the said random matrix. These can be found in 15 .

Assume $\lambda$ is a real eigenvalue of a fixed, real $n \times n$ matrix $A$. The true density of real eigenvalues, or the expected number of real eigenvalues per unit length can be defined:

$$
\rho_{n}(\lambda)=\left(\frac{1}{\sqrt{2 \pi}}\left[\frac{\Gamma\left(n-1, \lambda^{2}\right)}{\Gamma(n-1)}\right]+\frac{\left|\lambda^{n-1}\right| e^{-\frac{\lambda^{2}}{2}}}{\Gamma\left(\frac{n}{2}\right) 2^{\frac{n}{2}}}\left[\frac{\Gamma\left(\frac{(n-1)}{2}, \frac{\lambda^{2}}{2}\right)}{\Gamma\left(\frac{(n-1)}{2}\right)}\right]\right)
$$

or, in a different light:

$$
\rho_{n}(x)=\frac{d}{d x} E_{A} \#_{(-\infty, x)}(A)
$$

where $\#_{(-\infty, x)}(A) \equiv$ number of real eigenvalues of $A \leq x, E_{A}$ denotes the expectation value for a random $A$ and $\Gamma$ is the standard gamma function. Moreover the probability density of $\lambda_{n} \in R, f_{n}(\lambda)$ is given by:

$$
f_{n}(\lambda)=\frac{1}{E_{n}}\left(\frac{1}{\sqrt{2 \pi}}\left[\frac{\Gamma\left(n-1, \lambda^{2}\right)}{\Gamma(n-1)}\right]+\frac{\left|\lambda^{n-1}\right| e^{-\frac{\lambda^{2}}{2}}}{\Gamma\left(\frac{n}{2}\right) 2^{\frac{n}{2}}}\left[\frac{\gamma\left(\frac{(n-1)}{2}, \frac{\lambda^{2}}{2}\right)}{\Gamma\left(\frac{(n-1)}{2}\right)}\right]\right)
$$

or more simply:

$$
f_{n}(\lambda)=\frac{1}{E_{n}} \rho_{n}(\lambda)
$$

where $E_{n}$ denotes the expected number of real eigenvalues of the $n \times n$ random matrix.

Integrating $\rho_{n}$ along the real line provides the expected number of real eigenvalues. Edelman provides several formulas from such a calculation, the simplest being summarized by the asymptotic series given in corollary 5.2 of [15]]:

$$
E_{n}=\sqrt{\frac{2 n}{\pi}}\left(1-\frac{3}{8 n}-\frac{3}{128 n}+\frac{27}{1024 n^{2}}+\frac{499}{32768 n^{4}}+O\left(\frac{1}{n^{5}}\right)\right)
$$

Again, $E_{n}$ is the expected number for a real, $n \times n$ random matrix. The manner in which the convergence in measure is not absolutely continuous (with respect to Lebesgue measure) is highly relevant to our results - the manner in 
which the convergence is not absolutely continuous with respect to Lebesgue measure results in an expected density of real eigenvalues that is higher than other chords of length $2 \pi$. For a full discussion, see [15]. However, this is not a pathological problem since the distribution of real eigenvalues on the real line is uniform. Moreover, for our purposes, the expected value of the real eigenvalues is not enough since, if all the real eigenvalues are located at \pm 1 , then clearly there will exist many local bifurcations from fixed points due to purely real eigenvalues — and these bifurcations will likely be of high codimension. That such is not the case is given in the following corollary:

Corollary 1 (Corollary 4.5 [15]) If $\lambda_{n}$ denotes a real eigenvalue of an $n \times n$ random matrix, then as $n \rightarrow \infty$, the normalized eigenvalue $\frac{\lambda_{n}}{\sqrt{n}}$ converges in distribution to a random variable uniformly distributed on the interval [-1, 1]

Besides the results regarding the real eigenvalues, Edelman also provides information regarding the density of non-real eigenvalues:

Theorem 2 (Density of Non-Real Eigenvalues: Theorem 6.2 [13]) The density of a random complex eigenvalue of a normally distributed matrix is:

$$
\rho_{n}(x, y)=\sqrt{\frac{2}{\pi}} y e^{y^{2}-x^{2}} \operatorname{erfc}(y \sqrt{2}) e_{n-2}\left(x^{2}+y^{2}\right)
$$

where $e_{n}(z)=\sum_{k=0}^{n} \frac{z^{k}}{k !}$ and erfc $(z)=2 / \pi \int_{z}^{\infty}$ exp $\left.-t^{2}\right) d t$, the complementary error function. Integrating this over the upper half plane gives the number of non-real eigenvalues.

All of these results can be nicely concluded with the following two theorems regarding the circular law.

Theorem 3 (Theorem $6.3[\mathbf{1 3}]$ ) The density function $\hat{\rho}$ converges pointwise to a very simple form as $n \rightarrow \infty:$

$$
\lim _{n \rightarrow \infty} \frac{1}{n} \hat{\rho}(\hat{x}, \hat{y})= \begin{cases}\frac{1}{\pi} & \hat{x}^{2}+\hat{y^{2}}<1 \\ 0 & \hat{x}^{2}+\hat{y}^{2}>1\end{cases}
$$

where $\hat{\rho}_{n}$ is simply $\rho$ as a function of $\hat{x}=\frac{x}{\sqrt{n}}$ and $\hat{y}=\frac{y}{\sqrt{n}}$. Note that $\frac{\hat{\rho}(\hat{x}, \hat{y})}{n}$ is a randomly chosen normalized eigenvalue in the upper half plane.

Finally, Edelman's version of the circular law can be proved using theorem 3 and a central limit theorem.

Theorem 4 (Circular Law: Convergence in distribution [13]) Let $z$ denote a random eigenvalue of $A$ chosen with probability $\frac{1}{n}$ and normalized by dividing by $\sqrt{n}$. As $n \rightarrow \infty$, z converges in distribution to the uniform distribution on the disk $|z|<1$. Furthermore, as $n \rightarrow \infty$, each eigenvalue is almost surely non-real.

\section{RANDOM POLYNOMIALS AND COMPANION MATRICES}

Let us recall again why we are concerned with the special case of random polynomials; the linear part of the derivative of time-delay dynamical systems — the ones often used to fit real time-series data - are companion matrices. In particular, given the companion matrix:

$$
\left[\begin{array}{ccccccc}
a_{1} & a_{2} & a_{3} & \cdots & a_{d-2} & a_{d-1} & a_{d} \\
1 & 0 & 0 & \cdots & 0 & 0 & 0 \\
0 & 1 & 0 & \cdots & 0 & 0 & 0 \\
\vdots & \ddots & \vdots & & & & \\
0 & 0 & 0 & \cdots & 0 & 1 & 0
\end{array}\right]
$$

the corresponding characteristic polynomial - the equation whose solutions are the eigenvalues of the above matrix — is a polynomial as given in equation [15] Thus, the elements of a companion matrix (e.g. the $a_{k}$ 's) can be identified with the coefficients of the characteristic polynomial of the given matrix. The results we will discuss here briefly are from IVA while issues that arise due to computation of eigenvalues from companion matrices can be found in [16]. 


\section{A. Polynomials with Gaussian coefficients}

Let us begin with the polynomial

$$
a_{0}+a_{1} x+a_{2} x^{2}+\cdots+a_{n} x^{n}
$$

where the $a_{i}$ coefficients are independent standard normals with mean zero. The expected number of real zeros, $E_{\text {real }}$, as $n \rightarrow \infty$ is given by the formula:

$$
E_{\text {real }}(n)=\frac{2}{\pi} \log (n)+C_{1}+\frac{2}{n \pi}+O\left(1 / n^{2}\right)
$$

where $C_{1}=0.6257358072$ (cf. theorem 2.1 in [14] or [26]). This formula is calculated by integrating the true density which is given by:

$$
\rho_{d}(x)=\frac{1}{\pi} \sqrt{\frac{1}{\left(x^{2}-1\right)^{2}}-\frac{(d+1)^{2} x^{2 d}}{\left(x^{2 d+2}-1\right)^{2}}}
$$

Careful analysis of equation 17 yields the limiting density of real eigenvalues - that as $n \rightarrow \infty$, the density of real eigenvalues is concentrated at \pm 1 . The expected value of real roots is, of course, a crude measurement of interest as we are interested in differentiating between bifurcations due to real and complex eigenvalues. The density provides considerably more insight. Moreover, the convergence of the density will have a significant impact on the probability of the first bifurcation as we will see in section $\nabla 1$ where we compare equation 17 with the numerical results.

\section{Non-zero mean}

The random polynomials is the only case we will consider for which there are theoretical results regarding the convergence of the density of real zeros for distributions with non-zero means. The effect of a non-zero mean on the distribution of the coefficients of a random polynomial is neatly summarized by the following theorem:

Theorem 5 Consider a random polynomial of degree $d$ with coefficients that are independent and identically distributed normal random variables. Define $m \neq 0$ to be the mean divided by the standard deviation. Then, as $d \rightarrow \infty$,

$$
E_{\text {real }}(d)=\frac{1}{\pi} \log (d)+\frac{C_{1}}{2}+\frac{1}{2}-\frac{\gamma}{\pi}-\frac{2}{\pi} \log (|m|)+O(1 / d)
$$

where $C_{1}=0.6257358072 \ldots$ as previously defined, and $\gamma=0.5772156649 \ldots$ is Euler's constant. Furthermore, the expected number of positive zeros is asymptotic to

$$
\frac{1}{2}-\frac{1}{2} \operatorname{erf}^{2}\left(\frac{|m|}{\sqrt{2}}\right)+\frac{1}{\pi} \Gamma\left[0, m^{2}\right]
$$

The proof can be found in [14] (theorem 5.3). Considering equation 18 and comparing it with equation [16 one arrives at the comparison between $\sim \log (d)+\frac{1}{d}$ for zero mean with $\sim \log (d)-\log (|m|)$; as $d \rightarrow \infty$, the effect of the nonzero mean is a shift of the $\log (d)$ curve by $-\log (|m|)$. This dependence can be seen in Fig. 2

\section{B. Real zeros of random functions with random coefficients}

The goal of this paper is to consider the probability of the type of bifurcation from a fixed point in a class of neural networks that can be used to approximate time-series data from actual physical experiments. In [14] a remarkable theorem was proved regarding sums of differentiable functions with random coefficients linking the distribution to the coefficients and the function type to the distribution of real zeros. This theorem has a high degree of relevance to a study such as ours since it offers the hope of applying to the class of mappings we are considering.

Theorem 6 Let $v(x)=\left(f_{0}(x), \ldots, f_{n}(x)\right)^{T}$ be any collection of differentiable functions and $a_{0}, \ldots, a_{n}$ be the elements of a multivariate normal distribution with mean zero and covariance matrix $C$. The expected number of real zeros on an interval (or measurable set) I of the equation

$$
a_{0} f_{0}(x)+a_{1} f_{1}(x)+\cdots+a_{n} f_{n}(x)=0
$$




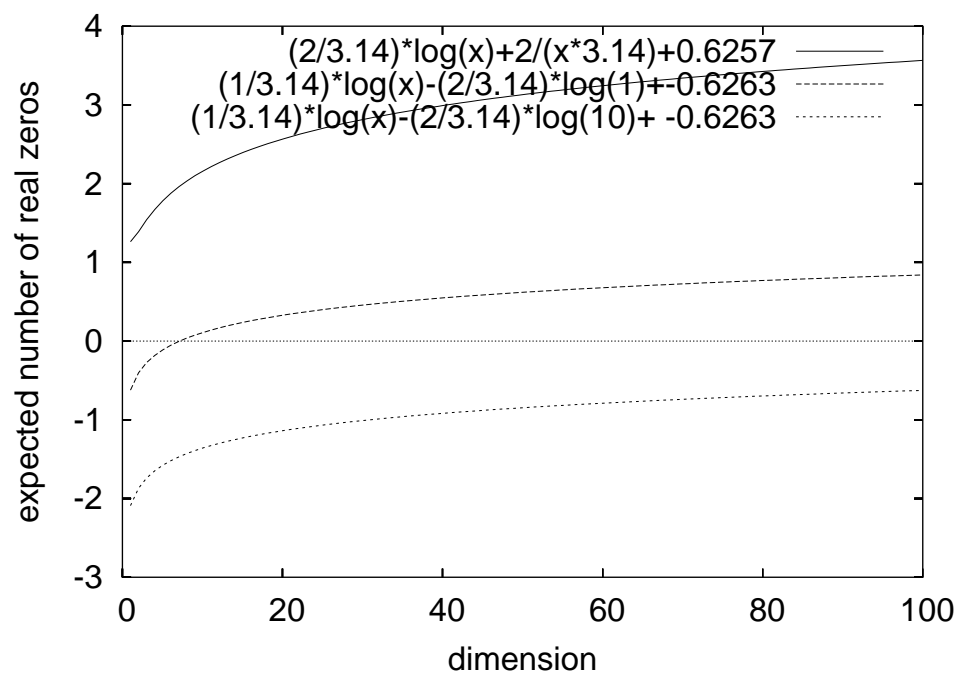

FIG. 2: A plot of equation 16 and 18 for $m=1,10$.

is

$$
\int_{I} \frac{1}{\pi}\left\|w^{\prime}(x)\right\| d x
$$

where $w$ is given by

$$
w(x)=\frac{C^{1 / 2} v(x)}{\left\|C^{1 / 2} v(x)\right\|}
$$

In logarithmic derivative notation this is

$$
\frac{1}{\pi} \int_{I}\left(\frac{\partial^{2}}{\partial x \partial y}\left(\left.\log \left(v(x)^{T} C v(y)\right)\right|_{y=x=t}\right)\right)^{1 / 2} d t
$$

There are many applications of this profound theorem presented in [14]. One of particular interest is an application to a trigonometric series such as

$$
\sum_{k=0}^{\infty} a_{k} \cos \left(\nu_{k} \theta\right)+b_{k} \sin \left(\nu_{k} \theta\right)
$$

where $a_{k}$ and $b_{k}$ are independent normal random variables with mean zero and variance $\sigma_{k}^{2}$. The density and, thus, the expected values of real zeros is quite easily computed given theorem [6] The density is constant and, therefore, the real zeros of the above random trigonometric sum are uniformly distributed on the real line. An important point to note is the sharp difference between the trigonometric case and the random polynomial case. Thus, different functional forms and distributions of $a_{k}$ 's give very different distributions of real zeros of polynomials. These two results show a sharp contrast regarding bifurcations from fixed points - the trigonometric series above is likely to yield all real bifurcations; whereas, the standard random polynomial case yields significantly more bifurcations due to complex eigenvalues. Providing a similar analysis for neural networks like those given in equation 3 is complicated by the sum inside the activation function and, therefore, is beyond the scope of this paper. However, as we will discuss later, because neural networks can approximate nearly any $C^{r}$ mapping which include both the random polynomials and the trigonometric series, altering the distributions of the $\beta_{i}$ 's and the $w_{i j}$ will clearly yield very different densities of real zeros. A relation between the distribution of the $\beta_{i}$ 's and $w_{i j}$ 's will be the focus of section VID.

\section{A CONJECTURE REGARDING THE FIRST BIFURCATION FROM A FIXED POINT}

There are three generic, codimension-one, local bifurcations from a fixed point in maps of dimension two or greater 29] 9]. These three bifurcations depend on symmetries of the dynamical system, but generally they consist of: the 
flip bifurcation, corresponding to the largest eigenvalue being -1 ; the fold, corresponding to the largest eigenvalue being 1; and the Neimark-Sacker [31] [36], corresponding to a complex conjugate pair of eigenvalues with modulus one. Edelman, Girko, and Bai have all shown that in the infinite-dimensional limit, a real matrix with elements selected from a real Gaussian distribution, the normalized eigenvalues will be distributed uniformly on the unit disk in the complex plane. Since the Neimark-Sacker bifurcation corresponds to the bifurcation via a complex conjugate pair of eigenvalues, a logical application of the circular law is to infinite-dimensional dynamical systems whose Jacobian matrix has elements whose distribution has a finite sixth moment. In this circumstance, the probability one bifurcation would seem to be a Neimark-Sacker bifurcation. A conclusion along these lines will prove incorrect as we will show in section VI The restriction of a finite sixth moment will turn out to be too weak because lower-order moments can affect quantities like the spectral radius or the eigenvalue with the largest modulus. Limiting ourselves to the case where the matrix has real Gaussian elements with mean zero and unit variance for which we have more restricted and detailed results will be fruitful. For example, if the real eigenvalues concentrate near 1 and -1 , we will run into problems, but Edelman (corollary (1)) has shown that this circumstance will not occur. Instead, the real eigenvalues will be distributed uniformly on the real axis. Results akin to the aforementioned result will prove necessary for arguments involving bifurcations. In the standard and general bifurcation sequence constructions, one would be concerned with a parameterized curve of matrices. In such a scenario the matrices would not be independent along the curve in general. Surmounting this obstacle is yet an open problem. However, in some special cases, like where the parameterized curve is linear and forms an interval in say, $R^{1}$, the difficulties are greatly reduced. Thus, we can make the following statement:

\section{Corollary 2 ( First bifurcation probability ) Given the dynamical system F}

$$
F\left(x_{t-1}\right)=x_{t}=\epsilon A x_{t-1}+\epsilon G\left(x_{t-1}\right)
$$

where $x_{t} \in R^{n}, \epsilon \in R, A \in R^{n^{2}}, a_{i j} \in N(0,1)$, and where $G\left(x_{t-1}\right)$ is a nonlinear $C^{r}$ ( $\left.r>0\right)$ mapping of $x_{t-1}$ which is of order 2 or higher. Thus $F\left(x_{t-1}\right) \cong \epsilon A x_{t-1}$ for $\epsilon$ small. Assume $F$ has a fixed point at $\epsilon=0$ and upon the increase of $\epsilon, F$ undergoes a local, codimension-one bifurcation. As the dimension of the dynamical system $F$ goes to infinity (i.e. given $\left.A \in R^{n^{2}}, n \rightarrow \infty\right)$, the probability that the first bifurcation will be of type Neimark-Sacker will converge to one.

Proof: This result follows trivially from the results of Edelman [13], [15] and Girko [19] [21] [23] [22] [20].

We can, with a little work, impose a measure on the set of dynamical systems for which this result holds via results of Edelman, the neural networks 44, and some standard arguments using measure theory. Upon doing so, one nontrivial issue is understanding what such a set of dynamical system would "look" like. We will refrain from further discussion of this extension here. It was originally hoped that we could extend this result such that the elements of the $A$ matrix can be selected from any distribution with a finite sixth moment in line with the circular law of Bai [8]. However, considering only random matrices with elements chosen from a Gaussian distribution, it can be shown numerically that the mean of the distribution has a significant effect on the eigenvalue with the largest modulus.

Corollary (2) falls far short of satisfying desires. First, corollary (2) does not speak to the probability of its hypothesis being satisfied in a general space of dynamical systems. Again, we can construct a measure such that the relevant hypothesis are always satisfied, but how general such a measure is, which is the same problem, is still a problematic issue. Moreover, corollary (2) is not cast in the general parameterized curves of the bifurcation theory framework we desire - linear "curves" are very limiting. See [39] or [4] for a construction of the general bifurcation framework to which we are referring. Providing an answer to the question regarding general spaces of $C^{r}$ mappings would likely require establishing some type of measure on the space of general $C^{r}$ dynamical systems, and establishing such a measure is often very difficult, to say the least. If such a measure could be defined, some meaningful notion of equivalence in measure would need to be shown. This issue, however, would likely be the least of the problems. Moreover, corollary (2) does not provide any information regarding how large, but finite-dimensional, dynamical systems that satisfy all the hypotheses behave. Lastly, corollary (2) does not provide any insight into how the convergence to such a result might occur as the dimension of the dynamical system is increased. Thus, we will present a conjecture that we believe captures more of what we want.

Conjecture 1 (Genericity of Neimark-Sacker bifurcations in high-dimensional dynamical systems) $B e$ gin with a space of $C^{r}(r>0)$ dynamical systems with bounded first derivatives whose elements form a distribution with zero mean on compact sets with a single real parameter and such that there exists a fixed point on a measurable interval of the parameter space, and at least one local bifurcation upon a continuous variation of the parameter. There exists a probability measure on the parameters such that, as the dimension d of the dynamical system is increased, the probability of the bifurcation from fixed points via the Neimark-Sacker bifurcation will increase and approach probability one as $d \rightarrow \infty$. 
Of course this conjecture makes the most sense in a framework such as that provided by neural networks for which the parameter space is large enough that it can have some approximation of $C^{r}$ function space. We will not discuss this conjecture further here, but upon the presentation of the numerical results, we will provide a discussion of what this conjecture might mean and where and how it is known to fail.

Finally, the above construction does not apply explicitly to the time-delay dynamics in general — whose linear derivative matrices are companion matrices — or to the neural networks we are focusing on in particular. Such a circumstance would require a slightly different conjecture:

Conjecture 2 (Genericity of Neimark-Sacker bifurcations in high-dimensional time-delay dynamical systems) Begin with the space of $C^{r}(r>0)$ time-delay dynamical systems with bounded first derivatives on compact sets with a single, real parameter and such that there exists a fixed point on a measurable interval of the parameter space, and at least one local bifurcation upon a continuous variation of the parameter. There exists a probability measure on the parameters such that, as the dimension $d$ of the dynamical system is increased, the probability of the bifurcation from fixed points via the Neimark-Sacker bifurcation will increase, and approach probability one as $d \rightarrow \infty$.

Analysis of this conjecture is akin to a study of random polynomials or a sum of random functions with coefficients drawn from a random distribution. This case is of interest because many experimental results come from time-series data - data that is reconstructed with a "universal approximator" that forms a time-delay dynamical system (neural networks of the type we consider fall into this class). We will compare and contrast the various results and implications of the constructions of the two conjectures. Because neural networks are universal approximators, we will, at the end, suggest how to find the measure on the $a_{k}$ 's that will link them to the general random matrix framework.

\section{NUMERICAL CASES}

We will begin our numerical investigations with a careful analysis of Gaussian random matrices with mean zero and variance 1 - one of the cases for which Edelman has provided theoretical results. We will then begin to investigate the difference between, and hence generality of, what we can imply regarding conjecture 1 with the results of Edelman versus those of Bai by perturbing the first and second moments of the Gaussian matrices and observe differences. Following this, we will study the case of uniform random matrices — a case for which Bai's results still apply — to begin to understand the invariance of various results to different random distributions. We will again study the perturbation of the first and second moments of the uniform distribution on the relevant quantities. Since the Jacobian of a time-delay dynamical system at a fixed point is a companion matrix, we will then briefly study companion matrices and finally move on to the case of time-delay neural networks.

\section{A. $M_{n}$ Gaussian}

As a base case, we will begin our analysis with matrices $G$ that have elements chosen from a Gaussian distribution with mean zero and variance one $(N(0,1))$. This is the case that both Girko and Edelman investigated, and thus there exist several theorems upon which we can verify our work. We will then begin perturbing various moments of this distribution to better understand the different implications and limitations of the work of Edelman, Girko, and Bai as applied to bifurcation theory.

\section{Gaussian matrices with zero mean}

Begin with the mean zero, variance one case, a comparison between Edelman's expected number of real eigenvalues given in equation 12 and those empirically calculated from random matrices is depicted in Fig. 3. The lines overlay nearly perfectly even at low dimensions yielding a power-law dependence of $E\left[\lambda_{\text {real }}\right]=0.978 d^{-0.529}$ as expected.

Given corollary 1 - that the distribution of real eigenvalues converges to a uniform distribution on the real line - a disproportionate fraction of the bifurcations should be due to complex eigenvalues especially as $d \rightarrow \infty$. Figure 4 depicts the fraction of bifurcations that correspond to Naimark-Sacker, flip, and fold bifurcations as well as the fraction of eigenvalues that are real. For systems we are considering, these distinctions are easily made by simply calculating the largest eigenvalue and determining whether it is complex or real, combined with the sign of the eigenvalue. The results are as one might expect in the sense that the number of real bifurcations due to positive and negative eigenvalues are nearly identical. However the fraction of real eigenvalues converges to zero considerably faster than the fraction of bifurcations due to real eigenvalues. Considering the right plot of Fig. 4 the spectrum of eigenvalues of a $1024 \times 1024$ matrix, the real line is clearly highly and evenly populated with $\sim 30$ real eigenvalues. 


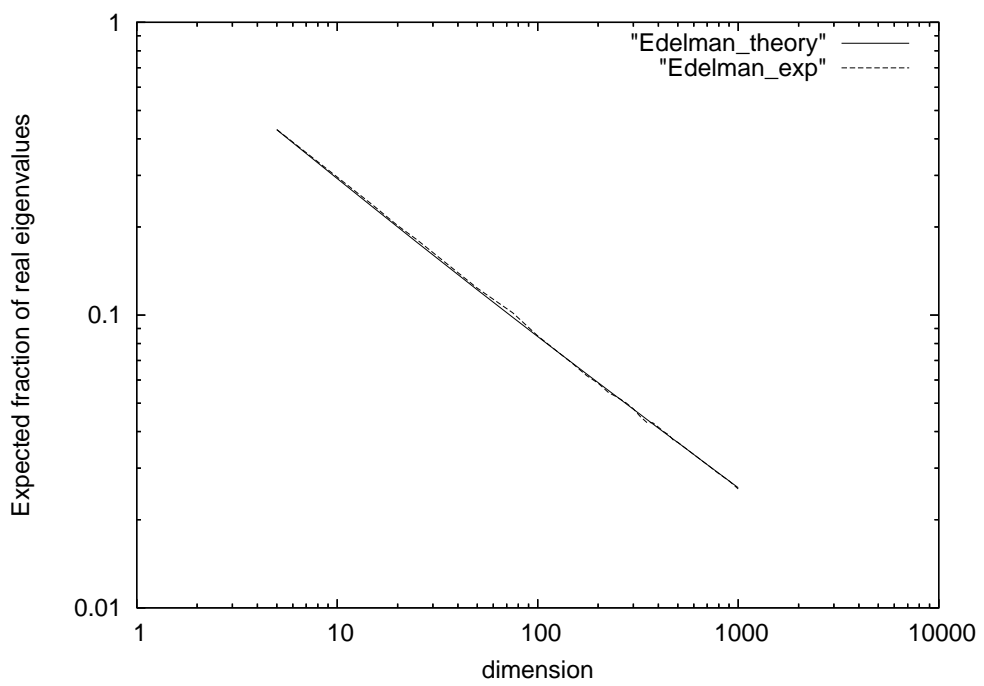

FIG. 3: Edelman's prediction for the expected fraction of real eigenvalues and the empirically calculated expected number of real eigenvalues. Both quantities were calculated in increments of 5 dimensions up to $d=50$ and then increments of 25 thereafter until $d=1000$. The line is that of a power law with $E_{\lambda_{\text {real }}}[d] \sim 0.9784 d^{-0.5291}$ as expected.
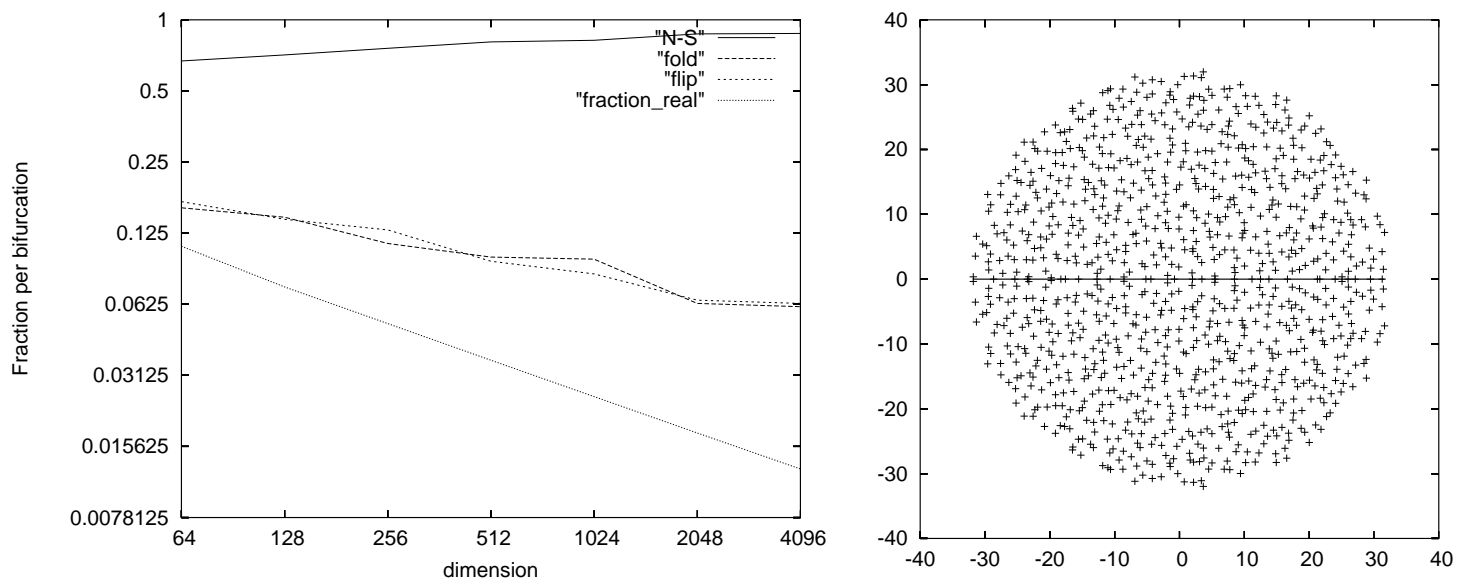

FIG. 4: On the left, the observed probability of each bifurcation was recorded for 1000 matrices with i.i.d., mean zero, variance one, Gaussian elements for each $d$ (in powers of 2) along with the fraction of eigenvalues that are real. On the right is the spectrum of eigenvalues in the complex plane that corresponds to a single $1024 \times 1024$ matrix $(d=1024)$.

Hence the convergence to uniformity seems to be well-behaved by $d=1024$. Nevertheless, the fraction of bifurcations due to real eigenvalues is clearly decreasing with $d$ in a power law. Moreover, the decrease of bifurcations due to 1 and -1 are nearly identical as is expected.

\section{Perturbing the first moment - Gaussian matrices with a non-zero mean}

The results of Girko and Edelman pertain to real random matrices with Gaussian matrices with mean zero and finite variance, while the results of Bai apply to any real random matrix with a distribution with a finite sixth moment. There is a great difference between these two formulations. Beginning with a standard Gaussian random matrix and perturbing the first moment (the mean), yields a result that has little relevance for the random matrix theory but has significant implications from the prospective of bifurcation theory. Summing a $d \times d$ matrix $G$ as a matrix with elements $g_{i j}$ drawn from a Gaussian distribution with mean zero and variance one $(N(0,1))$ and a constant $d \times d$ 


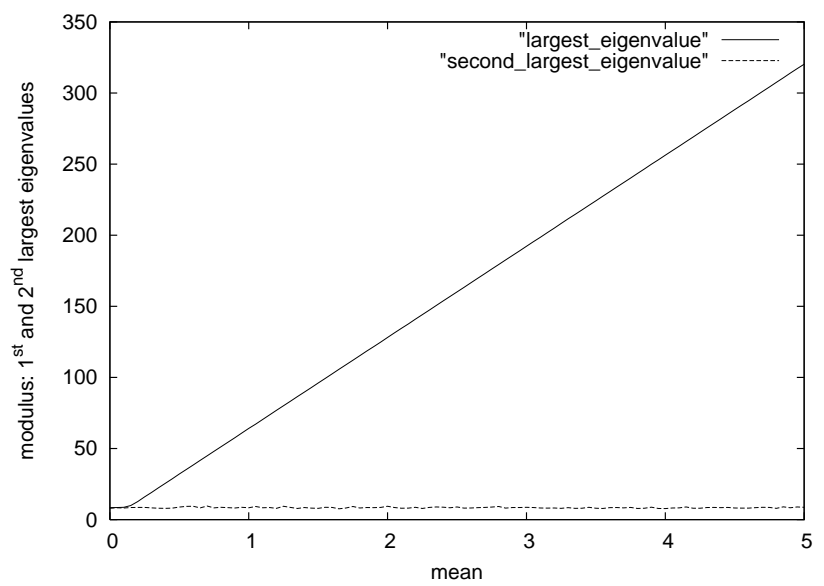

FIG. 5: This figure represents an ensemble of $1000 d \times d$ matrices with $d=64$. Depicted are the modulus of largest and second largest eigenvalues. The line representing the modulus of the largest eigenvalue is given by $64 \mathrm{~m}$ while the line for the modulus of the second largest eigenvalue is given by $\sim \sqrt{d}$.

matrix $P_{m}$ with elements $p_{i j}=m$ yielding

$$
\Lambda_{m}=G+P_{m}
$$

Figure 5 has the modulus of the largest and second largest eigenvalues plotted for $\Lambda_{m}$ with $d=64$. For $|m|>0.1289$, the largest eigenvalue is always real and increases with $m$ according to:

$$
\lambda_{d}(m)=d m
$$

The next largest eigenvalue is most often complex as would be expected if the distribution of eigenvalues on the unit disk is uniform. However, the modulus of the second largest eigenvalue(s) is constant independent of $m\left(\lambda_{d-1}=\right.$ constant) and is approximately $\sqrt{d}$ as expected from considering Edelman's normalization formulas. Thus, aside from the largest real eigenvalue, the distribution of eigenvalues behaves like the spectrum of $G$. Therefore, a dynamical system with a $D F$ matrix

$$
\frac{\epsilon}{\sqrt{d}+a} \Lambda_{m=\frac{1}{d}}
$$

for $a<<1$, upon increasing $\epsilon$, will always undergo a flip bifurcation from a fixed point. The point of this is that Bai's convergence of the spectrum to a uniform distribution on the unit disk in the complex plane of a random matrix with elements drawn from a distribution with finite sixth moment is not sufficient to guarantee that a dynamic system with a Jacobian matrix (at a fixed point) with elements that converge to a distribution with a finite sixth moment, will bifurcate as a matrix that was defined by having eigenvalues in a uniform distribution on the unit disk for all $d$. In this case, a measure-zero set (a single eigenvalue), happens to be of utmost importance if considering the most probable bifurcation from a fixed point. Moreover, this single eigenvalue is not a counter-example to Bai's theorem because aside from the one single eigenvalue, the rest of the spectrum converges uniformly on the unit disk in the complex plane.

\section{Perturbing higher moments}

Perturbation of the second moment of the distribution of $G$, which amounts to perturbing the variance, has a simple effect on the spectrum - the spectral radius increases linearly with the variance. This will have very little effect on the bifurcation structure aside from decreasing the $\epsilon$ value for which the first bifurcation from a fixed point will occur. Perturbing higher moments of the distribution and the subsequent effects remains an open problem.

\section{B. $M_{n}$ uniform}

Above we considered matrices with entries drawn from Gaussian distributions. As a result, we could bring to bear a large body of analytical machinery. Now, however, we turn to matrices with entries drawn from iid uniform random 

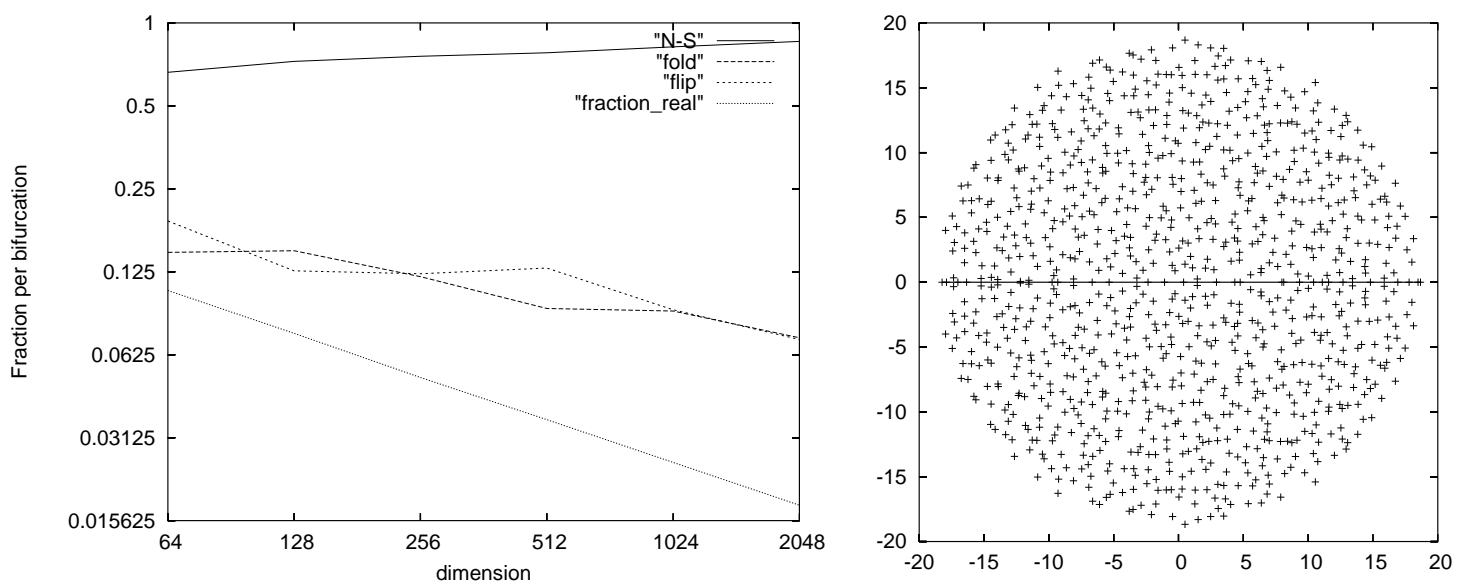

FIG. 6: On the left, the observed probability of each bifurcation was recorded for 1000 matrices with i.i.d. uniform elements for each $d$ (in powers of 2) along with the fraction of eigenvalues that are real. On the right is the spectrum of eigenvalues in the complex plane that corresponds to a single $1024 \times 1024$ matrix $(d=1024)$.

variables on the interval $(a, b)$. Unfortunately, in this circumstance, very little analytical machinery is available.

\section{Uniform matrices with zero mean}

For simplicity, we will set $a=-1$ and $b=1$ such that the $u_{i j}$ 's are i.i.d. uniform random variables on $(-1,1)$, with mean zero. Concentrating first on the fraction of real eigenvalues, Fig. 6]yields a scaling law of $E\left[\lambda_{\text {real }}\right]=0.919 d^{-0.517}$. This is similar to the theoretical and empirically calculated $E\left[\lambda_{\text {real }}\right]$ for a random matrix with Gaussian elements with zero mean. However, $E\left[\lambda_{\text {real }}\right]$ approaches zero faster in the case of Gaussian random matrices than with uniform random matrices. The difference is, nevertheless, quite small (about 3 percent). Accordingly, the fraction of real bifurcations decreases slightly slower for uniform random matrices than for the Gaussian case, while the fraction of flip and fold bifurcations are identical up to standard error. The right plot in Fig. [6 is indistinguishable from the analog plot for Gaussian random matrices given in Fig. 4. The real line is highly and evenly populated with $\sim 25$ real eigenvalues.

\section{Perturbing the first moment - Gaussian matrices with a non-zero mean}

The uniform random variable case is fundamentally different from the Gaussian case in several ways. First, the uniform case has finite support on $R$. Second, the mean is directly related to the endpoints of the support of the distribution, i.e. $\bar{x}=\frac{b+a}{2}$. Thus perturbing the mean from zero amounts to upsetting the symmetry of the end points of the support about zero. To investigate the effects of perturbing the mean we fix $b=1$ and vary $a$ from 0 to -1 . Or, more explicitly, given

$$
\Lambda_{a}=U+P_{a}
$$

with $u_{i j}$ uniform random variables on $(0,1)$ and with $p_{i j}=a \forall i, j, a$ is varied on $(-1,0)$. Figure $\mathbf{Z}$ depicts the dependence of the magnitude of the modulus of the two largest eigenvalues versus $a$ for a collection of $64 \times 64$ matrices. For $a=0$ to $a \sim-0.78$ the largest eigenvalue is real and its magnitude is given by

$$
\lambda_{d}(a) \sim-\frac{a}{4}
$$

The knee in the curve which occurs at $\sim-0.78$ for $d=64$ is dependent upon $b-$ the upper bound on the support of the distribution. Moreover, $\lambda_{d-1}$ does not remain constant with variation of the mean of the distribution but rather increases. However, aside from the largest eigenvalue, for $a \in(\sim-0.78,0)$, the distribution of eigenvalues appears uniform on a disk of radius $\left|\lambda_{d-1}\right|$. This effect is symmetric about zero. Fixing $a$ and varying $b$ will net the same effects. Thus, for $a \in(\sim-0.78,0)$, the corresponding dynamical system will have a 100 percent probability of undergoing a 


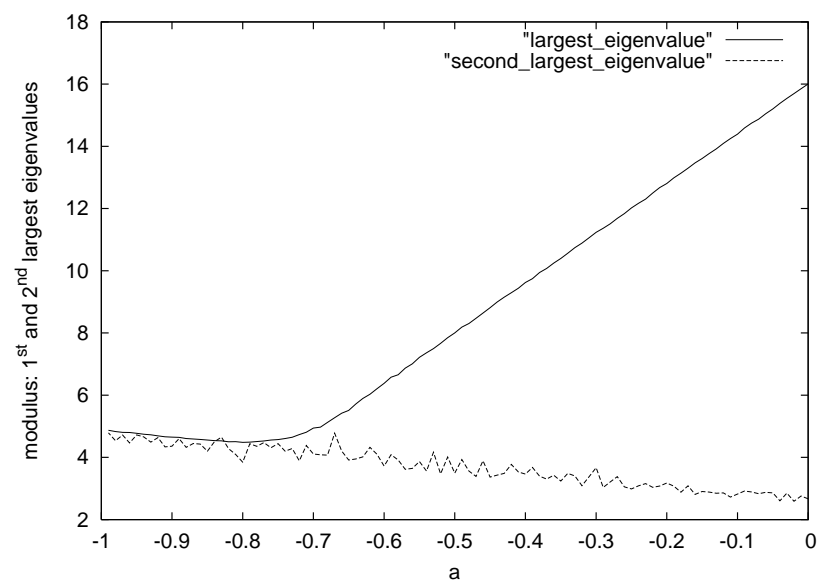

FIG. 7: This figure represents an ensemble of $1000 d \times d$ matrices with $d=64$ per $a$ increment where $\delta a=0.01$. Depicted are the modulus of largest and second largest eigenvalues. The line representing the modulus of the largest eigenvalue is given by $\frac{a}{4}$.

fold bifurcation. This result supports Bai's result because the convergence to uniformity of the eigenvalues on the unit disc is only violated by a single eigenvalue which is not relevant for random matrix results regarding distributions of eigenvalues. However, it is very relevant from our bifurcation theory prospective.

\section{Perturbing higher moments}

The variance of a uniform distribution on an interval $(a, b)$ is given by var $=\frac{(b-a)^{2}}{12}$. Thus, increasing the variance by a factor of $c$ while leaving the mean at zero is identical to multiplying $a$ and $b$ by $\sqrt{c}$, which is in turn identical to multiplying $u_{i j}$ by $\sqrt{c}$. Therefore, increases in the variance of a mean-zero uniform distribution of the elements of a random matrix $U$ has the effect of increasing the spectral radius by a factor of $\sqrt{c}$, which is simply a normalization factor and makes little difference to either the bifurcation prospective or the random matrix prospective. Again, perturbations of moments $>2$ is beyond the scope of this paper.

\section{C. $M_{n}$ companion Gaussian elements}

Beginning with Fig. 8 there are two important features. First, the eigenvalues appear to be distributed uniformly on the real line and on the unit circle, not on the entire disc. Second, the probability of a bifurcation due to a complex eigenvalue hovers near 55 percent over a range of $d=128$ to 1024 . This result, which seems surprising considering the spectrum depicted in Fig. 8 can be explained considering the convergence of the density of real zeros given by Edelman. This demonstrates how very important it is to consider the convergence of the densities when making claims regarding probable bifurcations.

Before we consider the densities, let us begin by considering the fraction of zeros that are due to real roots. Figure 9 portrays the numerically calculated fraction of real eigenvalues for a set of companion matrices with $a_{k}$ 's drawn from a normal distribution with mean zero and unit variance along with the predicted fraction of real zeros of Edelman. Clearly the two lines are in considerable agreement and have a power-law dependence with $\frac{E\left[\lambda_{\text {real }}\right]}{d}=1.52 d^{-0.831}$ for the numerically generated set and $E\left[\lambda_{\text {real }}\right]=1.78 d^{-0.849}$ as calculated from Edelman's formula given in equation [16 Note that the falloff of the fraction of zeros that are real is considerably faster than the other cases considered. This adds to the surprise found in Fig. 8 The fraction of eigenvalues that are real is decreasing like $d^{-0.85}$, yet the fraction of bifurcations due to real eigenvalues remains roughly constant for $d>128$.

This seeming contradiction, a decreasing fraction of real zeros with a constant fraction of bifurcations due to real eigenvalues, is rooted in the convergence of the density of real eigenvalues. Figure 10 shows both the empirical distribution (of 1000 polynomials) and the theoretical density of real zeros for polynomials of degree 64 with coefficients drawn from a Gaussian distribution with unit variance. The obvious spikes at \pm 1 are of interest, of course; however, it is the tails that extend above and beyond \pm 1 that have the most impact. This feature can be highlighted at $d=1024$ 

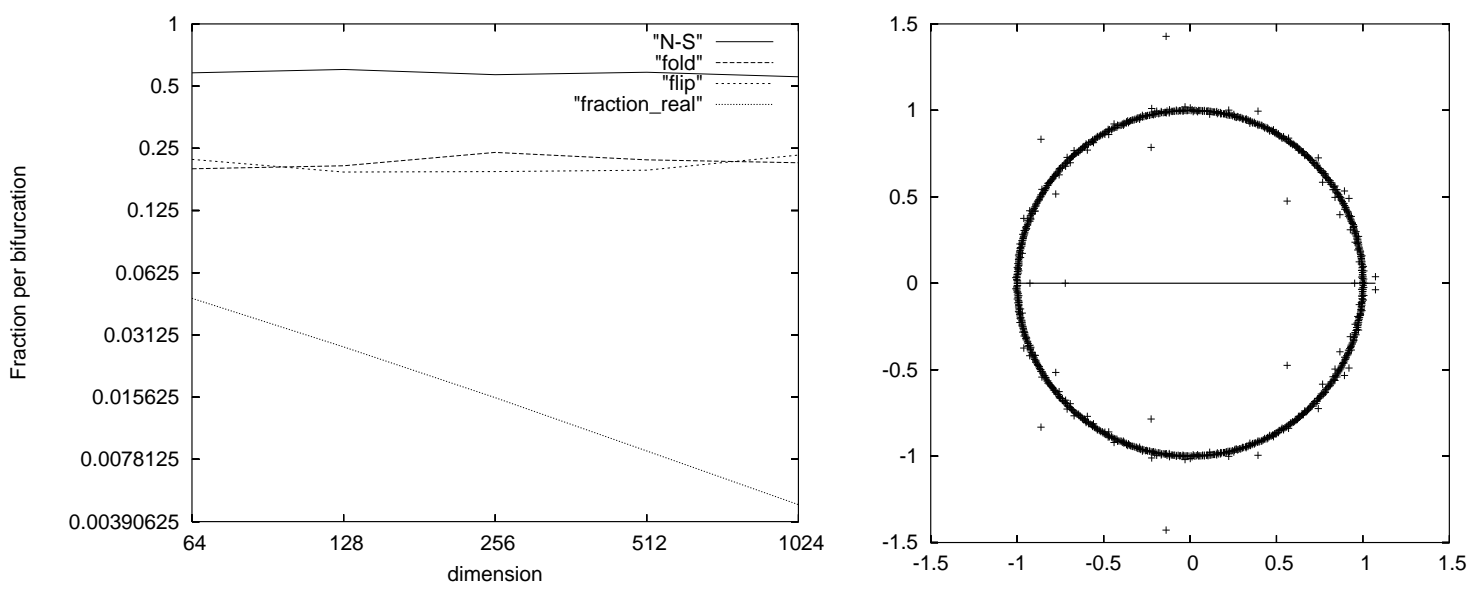

FIG. 8: On the left, the observed probability of each bifurcation was recorded for 1000 matrices with i.i.d., mean zero, variance one, Gaussian $a_{k}$ 's for each $d$ (in powers of 2) along with the fraction of eigenvalues that are real. On the right is the spectrum of eigenvalues in the complex plane that corresponds to a single $1024 \times 1024$ matrix $(d=1024)$.

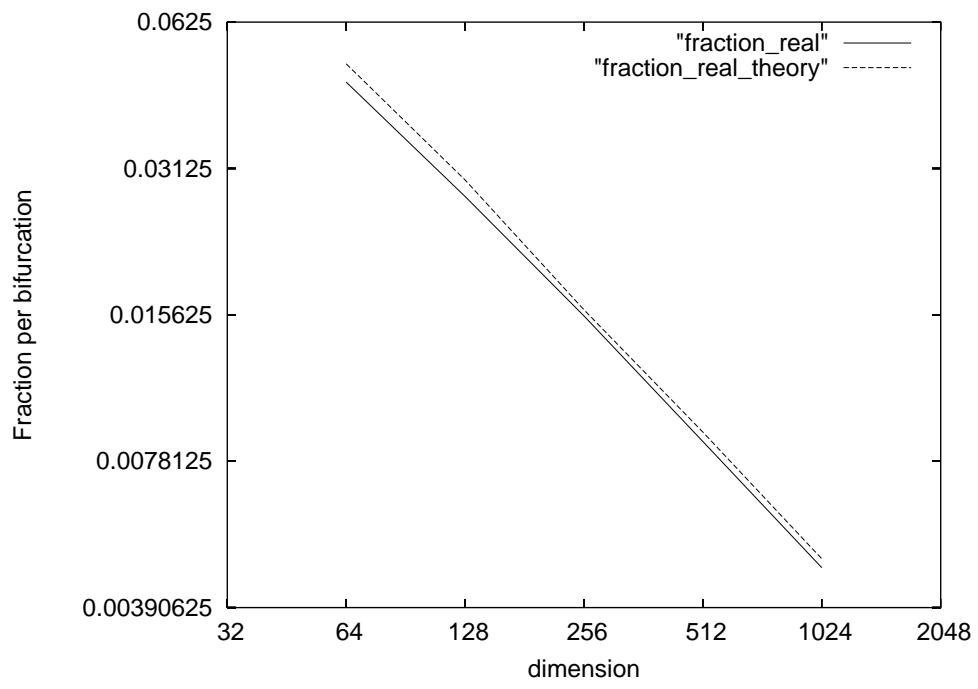

FIG. 9: Edelman's prediction for the expected number of real eigenvalues and the empirically calculated expected number of real eigenvalues. Both quantities were calculated for $d=64,128,256,512,1024$ and have a clear power-law dependence with $\frac{E\left[\lambda_{\text {real }}\right]}{d}=1.52 d^{-0.831}$ for the numerically generated set and $E\left[\lambda_{\text {real }}\right]=1.78 d^{-0.849}$ as calculated from Edelman's formula (equation 16).

if one considers Fig. 11] The point is that the complex eigenvalues exist largely on the unit circle while the real zeros have a non-zero measure set of zeros that have magnitude greater than one. The fraction of real bifurcations is largely determined by the convergence of the density of real eigenvalues - and because the convergence has a non-zero density with magnitudes greater than one, bifurcations due to real eigenvalues will persist to very high-dimensional matrices or polynomials. The fraction of real bifurcations is constant because the ratio of the measure of complex eigenvalues with magnitude greater than one to the measure of real eigenvalues with magnitude greater than one is relatively constant with increasing dimension.

\section{Neural Networks}

In the case of neural networks, we do not have the luxury of having a formula to guide our understanding of the empirical distribution of the real eigenvalues or the $a_{k}$ values. Compare Figs. 8 and 12 In a preliminary comparison 

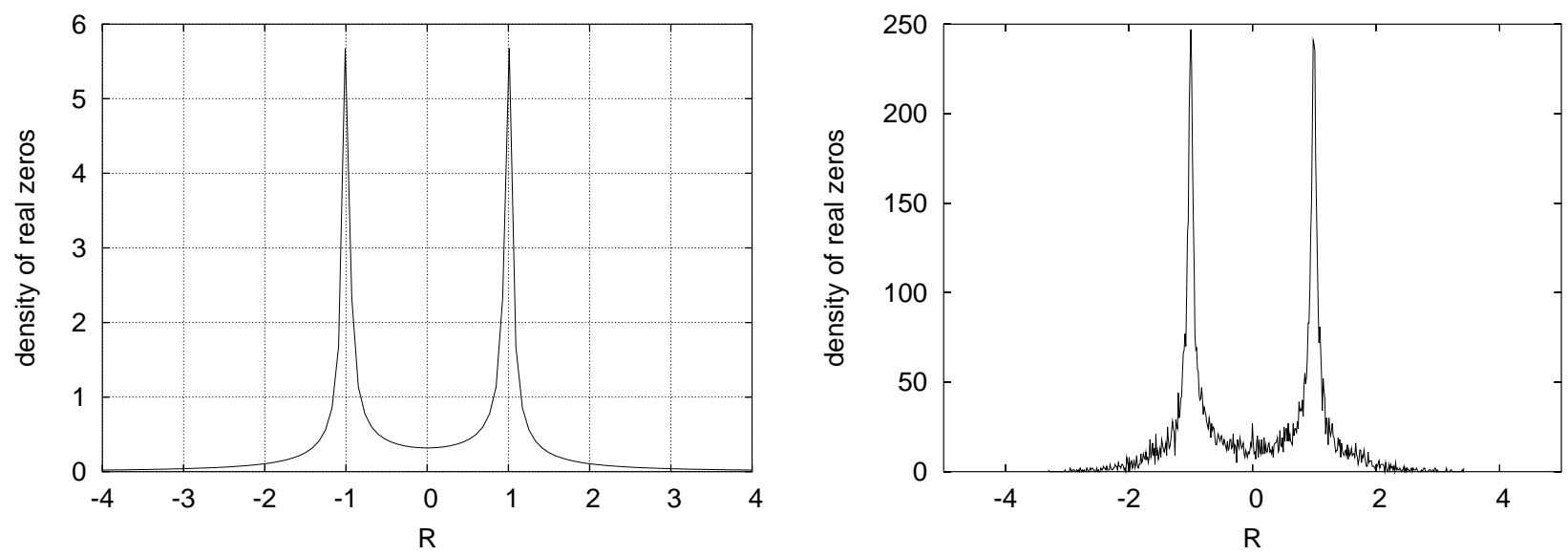

FIG. 10: On the left is the theoretical real zero density for a 64-degree polynomial with random coefficients drawn from normals with mean zero and unit variance. On the right is the real zero density for a set of 3000 companion matrices with $a_{k}$ 's drawn from standard normals with mean zero and unit variance.

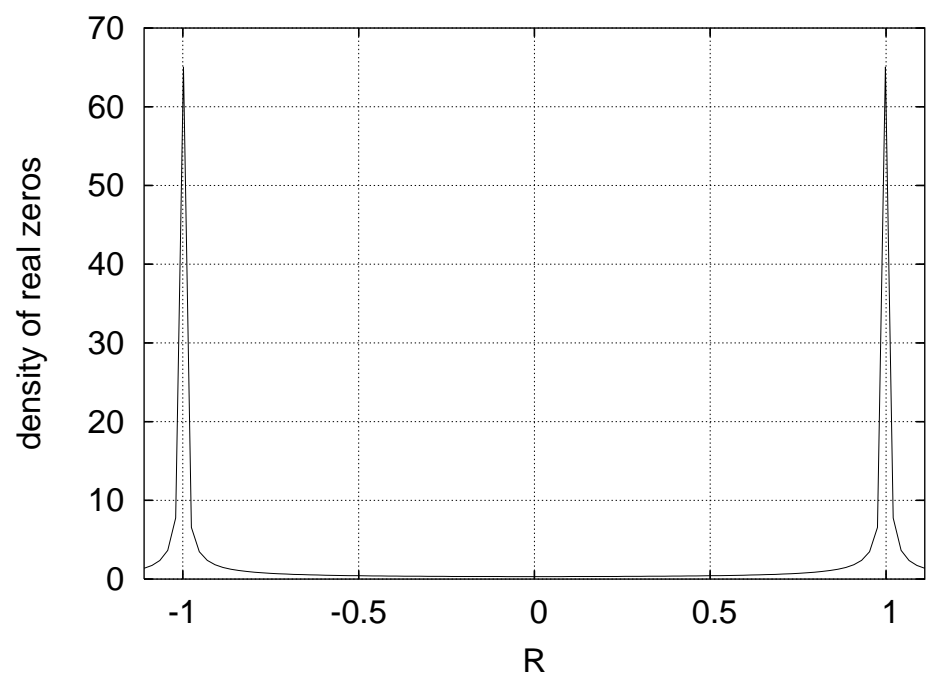

FIG. 11: Theoretical density of real zeros for polynomials of degree 1024 with coefficients drawn from a Gaussian distribution with mean zero and unit variance. For $|x|>1.12$ the density is zero.

between companion matrices with Gaussian $a_{k}$ 's and the neural networks with the weight structure (defined in section III), the distribution of eigenvalues appears nearly the same. However, the fraction of bifurcations of a fixed point that correspond to Naimark-Sacker type in the neural networks tends towards unity as the dimension tends toward infinity while the fraction of Naimark-Sacker bifurcations in the companion matrices with Gaussian $a_{k}$ 's tends toward a constant value $(\sim 0.58)$ as the dimension is increased. This difference can be rectified considering Fig. 13 which depicts the distribution of real zeros and the $a_{k}$ 's for the neural networks. With respect to the real zeros, adding dimensions has very little effect on the interval $(-0.9,0.9)$. However, near \pm 1 , the real eigenvalues are considerably more dense. However, there do not exist the tails above and below \pm 1 that are present in the companion matrices with Gaussian $a_{k}$ 's, which is enough to allow the fraction of bifurcations due to real zeros to tend to zero as the dimension is increased. At first glance, increasing $d$ has a significant effect on the variance $a_{k}$ 's at the first bifurcation. Because $s$ controls the variance of the $a_{k}$ 's this effect is due to the $s$ dependence of the first bifurcation. The $s$ dependence can be understood by considering Fig. 14] which characterizes the decrease in the mean $s$ at the first bifurcation. The decrease in the mean $s$-location of the first bifurcation obeys the power law $\sim a d^{0.63}$ where $a$ depends on $n$. Thus, with increasing $d$, the variance of the $a_{k}$ 's at the first bifurcation point will decrease. In general, the number of neurons has a negligible effect on the distribution of real eigenvalues and only a relatively minor effect on the peak of 

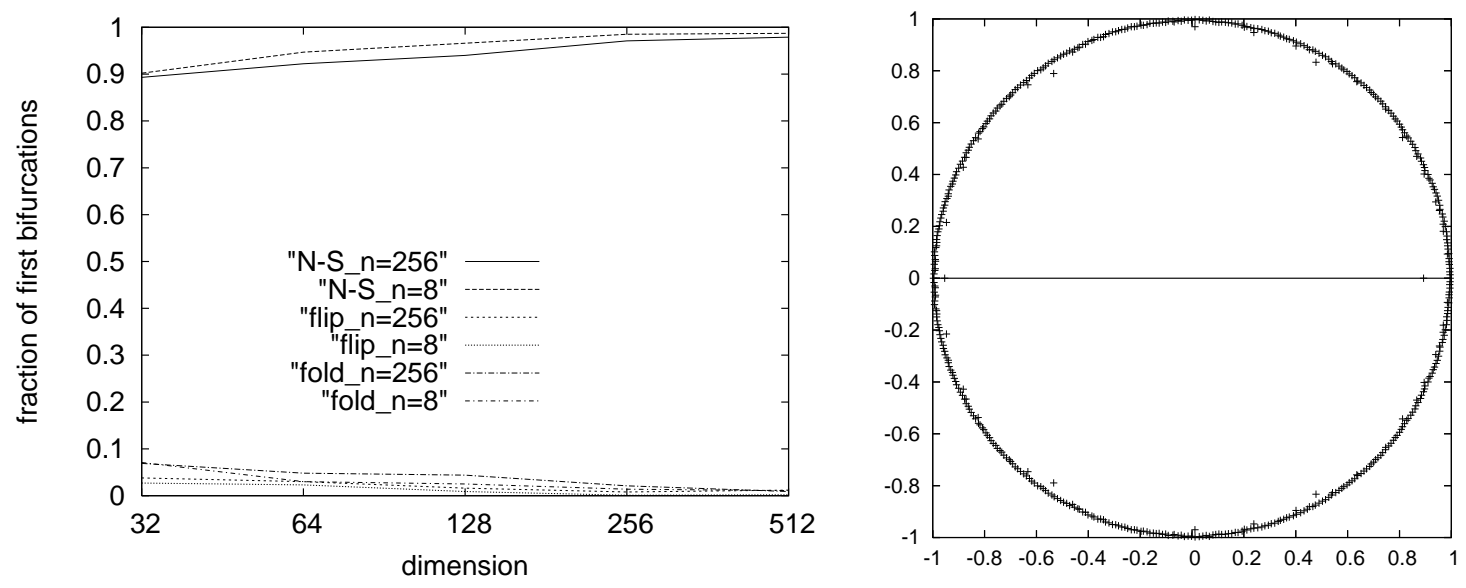

FIG. 12: On the left, the observed probability of each bifurcation was recorded for 1000 neural networks for each $d$ along with the fraction of eigenvalues that are real. On the right is the spectrum of eigenvalues in the complex plane that corresponds to a single neural network with $n=256$ and $d=512$.
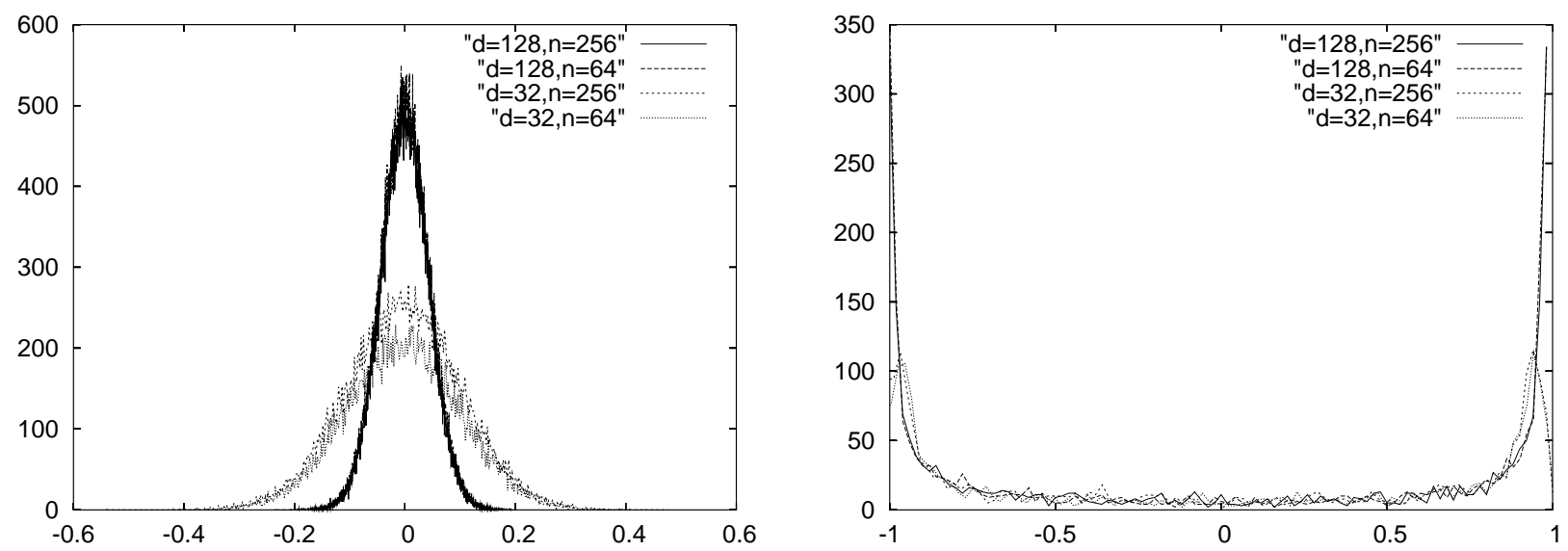

FIG. 13: The plot on the right is of the distribution of $a_{k}$ 's for 1000 neural networks with $n=256,64$ and $d=128,32$. The plot on the right is of the distribution of real eigenvalues along the real axis for the same set of neural networks.

the $a_{k}$ 's.

\section{REVISITING THE CONJECTURE}

The measure provided by Bai on random matrices — any distribution with a finite sixth moment — is quite general; it was originally hoped that such a measure would be enough to qualify probable bifurcations from fixed points in a large set of dynamical systems. As is now clear, simply perturbing the first moment of the distribution, while having no effects upon Bai's results, completely alters the probably of a bifurcation. In an intuitive sense, the difference corresponds to dynamical systems whose most slowly contracting directions are rotations versus dynamical systems for which a single, non-rotational contraction dominates the slowly contracting dimension. What we are left with is a much more complicated picture. It is likely, given our numerical results, that corollary 2 can be generalized to distributions with finite sixth moment and zero mean; however, we have not attempted to do so. Conjecture 1 is in part justified by the random matrix results presented in section $\mathbf{D}$ but clearly there is much room for a more complete numerical and analytical study. The apparent distribution independence in the standard random matrix case is not present in the companion matrix case. Simply considering the examples given by Edelman or the ones presented in the paper are enough to demonstrate the existence of the diversity in the eigenvalue spectrum with changes in the measure imposed on the $a_{k}$ 's. While the analysis of the Gaussian $a_{k}$ 's yields negative evidence for conjecture 2 the 


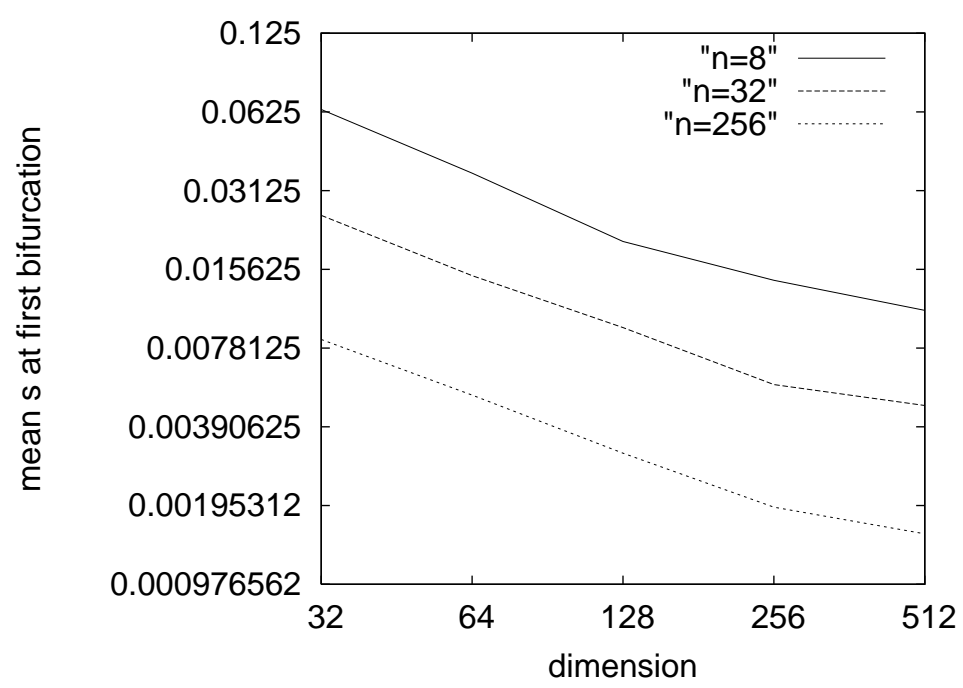

FIG. 14: The mean location of $s$ at the first bifurcation for $n=8, n=32$, and $n=256$ for networks of dimensions varying from 32 to 512 .

neural network analysis yields an example of a distribution of $a_{k}$ 's that supports conjecture 2] The constraints that are required of a distribution of $a_{k}$ 's that will yield adherence to conjecture 2 are to the authors completely unknown. In preliminary studies, One property that seems important for 2 to be valid is bounded distributions.

Comparison of the full random matrix and companion matrix cases is striking; the eigenvalue distributions for full random matrices are distributed on the unit disc as opposed to the unit circle in the case of companion matrices. However, there is, via the neural network approximation scheme, a very strong connection between the these two situations that has yet to be found. Recalling the discussion in section एA begin with a dynamical system

$$
F\left(x_{t-1}\right)=x_{t}=\epsilon A x_{t-1}
$$

where $\epsilon \in R$ is small enough such that $F$ is a fixed point, $A$ is a $d \times d$ real random matrix with Gaussian elements with mean zero and unit variance. There exists a neural network $f$ of dimension $2 d+1$ that can be trained on the time-series generated by $F$ such that $f$ will have a spectrum for which $d$ of it's eigenvalues will be identical (within the desired numerical accuracy) as well as $d+1$ free eigenvalues that will have magnitude less than one. Despite how the $d+1$ "free" eigenvalues are distributed, the remaining $d$ eigenvalues will have a distribution that is not on the unit circle and thus significantly different than any situations we have presented or know about. This connection yields insight into the distributions of $a_{k}$ 's of companion matrices (and thus the characteristic polynomial), as well as, forging a connection between standard dynamical systems and general time-delay dynamics. Finally, the neural networks provide an opportunity for a connection between real-world systems and the abstract dynamical models many in the field in dynamical systems study via training, and an understanding how their weight distributions affect their spectra.

\section{BEYOND FIXED POINTS}

Extending this construction beyond bifurcations of fixed points to the routes to chaos offers considerable problems. There are two basic approaches. One involves reduction of bifurcations for periodic orbits to bifurcations of fixed points in appropriately chosen coordinates. The other involves studying products of matrices of derivatives of periodic orbits.

Regarding the reduction of bifurcations of periodic orbits, one major problem arises because bifurcations of periodic orbits must be understood well enough to be approximated and reduced to analysis of fixed points. To see some of the currently open problems see [29]. Likewise, see 7] for nice explanations of the various approximation schemes. Then the measures on the random matrices must be carried through the various approximations.

Taking products of random matrices might be a fruitful approach (see [1] for results and techniques along these lines). However, linking them to periodic orbits might be difficult.

Various authors have studied the routes to chaos computationally. Utilizing the neural network framework discussed here [4] conclude that the most likely route to chaos is a quasi-periodic one - however these results are likely subject 
to the measures imposed upon the weight matrices. Likewise, Doyon et. al. [11] 12], Cessac et. al. [10], and Sompolinsky et. al [38] have arrived at similar conclusions in a variety of circumstances. Clearly any results such as these will be subject to the same dependences on the measures imposed on the weight matrices as were present in the case of the bifurcation of a fixed point mentioned in the previous section.

\section{FINAL REMARKS}

In the context of a general dynamical system if (i) the Jacobian of the fixed point can be identified with a full random matrix and (ii) if the distribution of elements has zero mean, then, for the cases we considered (uniform and Gaussian), as the dimension approaches infinity, the probability of a Naimark-Sacker bifurcation approaches unity. However, the largest real eigenvalue in the aforementioned circumstances scales linearly with the mean of the distribution of the elements of the matrix. Therefore, if the mean is large, the most probable bifurcation will be due to a real eigenvalue (e.g. a flip or a fold bifurcation), regardless of the dimension. An analytical understanding of linear scaling of the largest real eigenvalue with the mean of the distribution is unknown. Aside from the effect of the mean on the largest real eigenvalue, the result of the probability of a Naimark-Sacker bifurcation increasing with dimension is quite independent of the distribution. This is because, for nearly all distributions of elements, real random matrices have a spectrum that converges to uniformity on the unit disc as the dimension of the matrix goes to infinity.

In the context of time-delay dynamical systems, the story is different. In this case, the Jacobian forms a companion matrix. If the companion matrix has elements drawn from a Gaussian distribution, the probability of a NaimarkSacker bifurcation saturates at $\sim 58$ percent as the dimension goes to infinity. This is due to tails in the distribution of real zeros. Numerical results with time-delay, feed-forward neural networks however, behave very differently. For neural networks, as the dimension goes to infinity, the probably of a Naimark-Sacker bifurcation goes to unity. For companion matrices, the first bifurcation probability is highly dependent on the distribution of the elements of the matrix. In general, the spectrum of a time-delay dynamical system lies on the unit circle and the real line, not the entire unit disc as in the case with full matrices. However, the distriution of real zeros can vary significantly from distribution to distribution. Providing a link between the time-delay case and the standard dynamical systems framework via neural network training is suggested as future work.

\section{ACKNOWLEDGMENTS}

We thank R. A. Bayliss, W. D. Dechert, W. Brock, J. Albers, J. Thompson, J. Jost, I. Dobson, C. McTague, and J. C. Crutchfield for helpful discussions. This work was partially supported at the Santa Fe Institute under the Networks Dynamics Program funded by the Intel Corporation and under the Computation, Dynamics, and Inference Program via SFI's core grants from the National Science and MacArthur Foundations. Computing was done primarily on the Max Planck Institute for Mathematics in the Sciences Beowulf cluster, however significant computations were carried out on the U.C. Davis Computational Science Beowulf cluster and the Santa Fe Institute Beowulf cluster.

[1] Random matrices and their applications, volume 50 of Contemoporary mathematics. AMS, 1986.

[2] R. Adams and J. J. F. Fournier. Sobolev Spaces. Elsevier, 2nd edition, 2003.

[3] D. J. Albers and J. C. Sprott. Structural stability and hyperbolicity violation in large dynamical systems. Submitted, http://arxiv.org/pdf/nlin.CD/0408011.

[4] D. J. Albers and J. C. Sprott. Routes to chaos in high-dimensional dynamical systems: A qualitative numerical study. submitted, 2005.

[5] D. J. Albers, J. C. Sprott, and W. D. Dechert. Routes to chaos in neural networks with random weights. Int. J. Bif. Chaos, 8:1463-1478, 1998.

[6] Shunichi Amari, Hiroshi Nagaoka, and Shun-Ichi Amari. Methods of Information Geometry. ranslations of Mathematical Monographs. AMS, 2001.

[7] D. K. Arrowsmith and C. M. Place. An introduction to dynamical systems. Cambridge University Press, 1990.

[8] Z. D. Bai. Circular law. Ann. Probab., 25:494-529, 1997.

[9] P. Brunovsky. On one parameter families of diffeomorphisms. Commentationes mathematicae Universitatis Carolinae, 11:559-582, 1970.

[10] B. Cessac, B. Doyon, M. Quoy, and M Samuelides. Mean-field equations, bifurcation map and route to chaos in discrete time neural networks. Physica D, 74:24-44, 1994.

[11] B. Doyon, B. Cessac, M. Quoy, and M. Samuelides. "Control of the Transition to Chaos in Neural Networks with Random Connectivity". IJBC, 3:279-291, 1993. 
[12] B. Doyon, B. Cessac, M. Quoy, and M. Samuelides. On bifurcations and chaos in random neural networks. Acta Biotheo., 42:215-225, 1994.

[13] A. Edelman. The probability that a random gaussian matrix has $k$ real eigenvalues, related distributions, and the circular law. J. Multivariate Anal., 60:203-232, 1997.

[14] A. Edelman and E. Kostlan. How many zeros of a random polynomial are real? Bull. Amer. Math. Soc., 32:1-37, 1995.

[15] A. Edelman, E. Kostlan, and M. Shub. How many eigenvalues of a random matrix are real? J. Amer. Math. Soc., 7:247-267, 1994.

[16] A. Edelman and H. Murakami. Polynomial roots from companion matrix eigenvalues. Math. Computation, 64:763-776, 1995.

[17] R. Gencay and W. D. Dechert. An algorithm for the $n$ Lyapunov exponents of an $n$-dimensional unknown dynamical system. Physica D, 59:142-157, 1992.

[18] J. Ginbre. Statistical ensembles of complex, quaternion and real matrices. J. Math. Phys., 6:440-449, 1965.

[19] V. Girko. Circular law. Theory Probab. Appl., 29:694-706, 1984.

[20] V. Girko. Theory of random determinants. Kluer Academic, 1990.

[21] V. Girko. The circular law: ten years later. Random Oper. and Stoch. Eqns., 2:235-276, 1994.

[22] V. Girko. Strong circular law. Random Oper. and Stoch. Eqns., 5:173-196, 1997.

[23] V. Girko. The V-density of eigenvalues of non-symmetric random matrices and rigorous proof of the strong circular law. Random Oper. and Stoch. Eqns., 5:371-406, 1997.

[24] K. Hornik, M. Stinchocombe, and H. White. "Mulitlayer Feedforward Networks are Universal Approximators". Neural Networks, 2:359-366, 1989.

[25] K. Hornik, M. Stinchocombe, and H. White. "Universal Approximation of an Unknown Mapping and its Derivatives Using Multilayer Feedforward Networks". Neural Networks, 3:551, 1990.

[26] M. Kac. On the average number of real roots of a random algebraic equation. Bull. Amer. Math. Soc., 49:314-320 and 938, 1943.

[27] H. Kantz and T. Schreiber. Nonlinear Time Series Analysis. Cambridge University Press, $2^{\text {nd }}$ edition, 2003.

[28] A. Katok. Lyapunov exponents, entropy, and periodic orbits for diffeomorphisms. Publ. Math. I.H.E.S., 51:137-174, 1980.

[29] Y. Kuznetzov. Bifurcation Theory. Springer-Verlag, $2^{\text {nd }}$ edition, 1998.

[30] M. L. Mehta. Random Matrices, chapter 15. Elsevier, 2004.

[31] J. Neimark. On some cases of periodic motions depending on parameters. Dokl. Acad. Nauk SSSR, 129:736-739, 1959.

[32] V. I. Oseledec. A multiplicative ergodic theorem. Lyapunov characteristic numbers for dynamical systems. Tras. Moscow Math. Soc., 19:197-221, 1968.

[33] W. Ott and J. Yorke. Learning about reality from observations. SIAM J. Applied Dynamical Systems, 3:297-322, 2003.

[34] Ya. B. Pesin. Lyapunov characteristic exponents and smooth ergodic theory. English transl. Russian Math. Surveys., 32:55-114, 1977.

[35] D. Ruelle. Characteristic exponents and invariant manifolds in Hilbert space. Ann. of math., 115:243-290, 1982.

[36] R. Sacker. On invariant surfaces and bifurcation of periodic solutions of ordinary differential equations. Technical Report 333, New York State University, 1964.

[37] T. Sauer, J. Yorke, and M. Casdagli. Embedology. J. Stat. Phys., 65:579-616, 1991.

[38] H. Sompolinsky, A. Crisanti, and H. J. Sommers. Chaos in random neural networks. Phys. Rev. Lett., 1988.

[39] J. Sotomayor. Structural stability and bifurcation theory. In Dynamical Systems, pages 549-560, 1973.

[40] J. C. Sprott. Chaos and Time-series Analysis. Oxford University Press, 2003.

[41] F. Takens. Detecting atrange attractors in turbulence. In D. Rand and L. Young, editors, Lecture Notes in Mathematics, volume 898, pages 366-381, Dynamical Systems and Turbulence, Warwick, 1981. Springer-Verlag, Berlin.

[42] S. Wiggins. Global bifurcations and chaos: Analytical methods. AMS. Springer-Verlag, 1988.

[43] In chapter 15 of Mehta's famous book one can find the words "An ensemble of matrices whose elements are complex, quaternion, or real numbers, but with no other restrictions as to their Hermitian or unitary character, is of no immediate physical interest, for their eigenvalues may lie anywhere on the complex plane." He later asserts that these matrices are, nevertheless, of interest in their own right.

[44] In theory we can impose a measure on the weights of the neural networks that mimics the results of random matrices, however we have not found this said measure. It is likely that it could be determined via training of neural networks on data from dynamical systems with random $(N(0,1))$ linear parts. 